\title{
LA MANAGERIALIZACIÓN DEL AULA: la gramática del rendimiento desde las narrativas de los estudiantes
}

\author{
Lorena Ramírez-Casas del Valle \\ Pontificia Universidad Católica de Valparaíso \\ Enrique Baleriola \\ Universitat Oberta de Catalunya \\ Vicente Sisto \\ Pontificia Universidad Católica de Valparaíso \\ Verónica López \\ Pontificia Universidad Católica de Valparaíso \\ Felipe Aguilera \\ Pontificia Universidad Católica de Valparaíso
}

\begin{abstract}
Resumen
En Chile prevalece una política de gestión basada en la rendición de cuentas e inspirada en las ideas del New Public Management. Bajo este marco se ha instalado una gramática centrada en el rendimiento académico con altas consecuencias para los distintos actores de la comunidad educativa, desde la cual se configura el quehacer en la escuela, así como también performance el comportamiento de los estudiantes. El propósito de este estudio es analizar las narrativas de los estudiantes respecto de su experiencia en un aula que se configura bajo esta gramática. El enfoque metodológico es cualitativo, en el cual se realizó entrevistas individuales y grupales a 59 adolescentes pertenecientes a dos escuelas públicas en Chile. Desde el análisis de contenido- discursivamente orientado- se presentan como resultados cuatro narrativas principales: 1) En el aula hay que ser buen estudiante, 2) No todos somos iguales en el aula, 3) La justificación de las sanciones en pro del rendimiento, y 4) No se puede perder tiempo con los otros. Se discuten los resultados a la luz de los nuevos cambios en las políticas educativas que buscan avanzar hacia una educación más inclusiva y democrática.

Palavras clave: Educación; Management Público; Estudiantes; Narrativas;
\end{abstract}

\begin{abstract}
In Chile prevails management of policy based on the new public management logic and the accountability model. Under this framework, a grammar focused on academic performance has been installed with high consequences for the different actors of the educational community, from which the work in the school, as well as the performance of the students' behavior, is configured. The purpose of this study is to analyze the narratives of the students regarding their experience in the classroom that is configured under this grammar. The methodological approach is qualitative, in which both individual and group interviews were conducted with 59 children belonging to two public schools in Chile. From a content analysis - discursively oriented - four main narratives are presented as results: 1) In the classroom you have to be a good student, 2) Not all of us are equal in the classroom, 3) The justification of sanctions for a good personal performance, and 4) Time cannot be wasted with others. The results are discussed with regard to the new changes in educational policies that seek to move towards more inclusive and democratic education.
\end{abstract}

Keywords: Education; Public Management; Students; Narratives. 
La Managerialización del aula: la gramatica del rendimento (...) 
En las últimas décadas somos testigos de cómo la lógica de la empresa privada ha adquirido fuerza en el ámbito de lo público en distintos países, esto, bajo la promesa de la mayor eficiencia y calidad educativa (GEWIRTZ; BALL, 2000; GEWIRTZ, 2002; GUNTER, 2008; RANSON, 2008). Es así, que en distintos ámbitos tales como la salud, el mundo del trabajo, y la educación, actualmente están siendo gestionadas desde el modelo conocido como nuevo management público, el cual opera bajo un enfoque de rendición de cuentas (accountability) (FALABELLA, 2014; FALABELLA; OPAZO, 2014; SISTO, 2011)

Chile es reconocido como un caso emblemático en la implementación de este modelo en el ámbito de la educación, en el cual desde la década de los años 80, bajo la dictadura de Pinochet, se promulgó una nueva constitución, así como también decretos legislativos que fortalecieron una gobernanza neoliberal, bajo la cual ésta se entiende como un servicio que debe dar respuestas a las personas, y asegurar a los consumidores, entiéndase las familias, los mejores resultados en los estudiantes (ASSAEL et al., 2011; RANSON, 2008; SISTO, 2011).

A pesar de la vuelta a la democracia ocurrida en los años 90, este modelo se ha fortalecido, pues en la actualidad, la efectividad escolar articula una parte importante de las políticas educativas en el país, desde la cual se organiza el sistema educativo y su financiamiento en base a tres términos claves como son: el desempeño, la evaluación y rendición de cuentas (accountability), muy acorde a los mandatos de organismos internacionales como el Banco mundial y la OCDE (SISTO, 2019). En este sentido, como señala Ranson (2008), se ha instalado una gramática centrada en el rendimiento académico, la cual es asumida como la clave de la calidad de la educación, cuya principal forma de medir se realiza a través de pruebas estandarizadas como medio de verificación y de rendir cuentas (ANDERSON et al., 2012).

Este modelo de rendición de cuentas ha repercutido en las comunidades educativas, modificando las metas institucionales y el ethos escolar, transformando con ello los roles de cada miembro del sistema educativo, generando así una profunda transformación ética en la forma de entender la enseñanza y la experiencia del aprendizaje (FALABELLA, 2014; GEWIRTZ, 2002; BALL, 2003; NIESCHE, 2015). Un claro ejemplo de esto es que actualmente la enseñanza y los aprendizajes en el aula muchas veces se focalizan en la preparación de los estudiantes para que logren buenos desempeños en la prueba nacional de medición de la calidad educativa conocida como SIMCE (Sistema de Medición de Calidad Escolar) (ASSAÉL, et al., 2011), dejando de lado otros aprendizajes que no son medidos en este tipo de evaluaciones.

Es bajo la primacía de los estándares de logros y la dependencia de los subsidios estatales asociados a los buenos resultados, que actualmente, ha permeado en el imaginario de la sociedad la idea de que el aprendizaje académico es necesariamente un asunto individual y privado (LUNA-FIGUEROA, 2015). Esta afirmación sigue la línea de los análisis que Terreni, Grinberg y Pérez (2017) y Grinberg (2011) hacen sobre la emergencia de un nuevo tipo de sociedad, las que denominan sociedades del gerenciamiento, entendidas como aquellas en las que prevalece como discurso el empresario de sí mismo, esto como oda a la 
individualidad y al mérito personal. En ellas, se entiende que la suma de los individuos es igual a la adición del esfuerzo de múltiples individualidades con intereses particulares, y no a un todo o un actor global que obedece al bien común.

Estas sociedades del gerenciamiento serían parte de lo que Gewirtz (2002) denomina la revolución del mercado, lo cual "no es sólo un cambio de estructura e incentivos. Es un proceso de transformación que pone en juego un nuevo conjunto de valores y un nuevo entorno moral" (GEWIRTZ, 2002, p.47). Esto ha implicado un cambio desde una cultura integral a una justificación instrumental de la eficiencia, cuya preocupación central es la competencia individual, los indicadores de eficiencia y rendimiento, en detrimento de un aprendizaje contextualizado y cooperativo (FALABELLA, 2014), que va permeando en los distintos actores de la escuela. De esta manera, esta gramática de la eficiencia y rendición de cuentas va moldeando las disposiciones de cada uno de los miembros de la escuela, naturalizando ciertas formas de percibir, juzgar, imaginar y actuar, e instalando referentes relevantes para evaluar la propia experiencia (RANSON, 2003; 2008). Se entiende entonces, que estas políticas conllevan un poder discursivo-performativo, que transforma los modos en que los actores, entre ellos los estudiantes, constituyen lo que es bueno y deseable en la escuela (BALL, 2006; GEWIRTZ, 2002), así como también performan sus comportamientos (LUNA-FIGUEROA, 2015). En este sentido, en los niños se configura una subjetividad individual, donde se diluye una "trayectoria vital permeada por múltiples condiciones culturales, sociales, económicos... en pro de un hazte a ti mismo" (GRINBERG, 2011, p.67), lo que fomenta el discurso meritocrático y el círculo vicioso de la competencia y la individualidad.

Si bien el análisis de los cambios y nuevos valores que imponen estas políticas han sido estudiados en relación a las nuevas configuraciones en la identidad docente (FARDELLA; SISTO, 2015), o respecto del agobio y la tensión en las que viven los docentes por la responsabilidad de tener que conseguir buenos resultados, o por la estigmatización que han sufrido las escuelas (ASSAÉL et al., 2014), así como también, la excesiva focalización del quehacer diario en las escuela por tener que conseguir las evidencias como forma de responder a la política (FALABELLA, 2014), se advierte menor desarrollo de investigaciones que aborden los efectos en los adolescentes desde sus propias experiencias en la escuela.

En este trabajo entenderemos joven como un actor social, lo cual supone el surgimiento de nuevas maneras de comprender la subjetividad y los procesos objetivos de su despliegue, no a partir de principios universales sino de experiencias vividas por los propios sujetos (PEÑA, 2004). En este sentido, nos interesó conocer cómo los estudiantes pertenecientes a establecimientos públicos que cursan séptimo año de educación básica construyen las narrativas de su experiencia en el aula, bajo la gramática del rendimiento y rendición de cuentas. Para esto, en una primera parte del escrito presentamos en detalle el marco legal y político que sustenta el rendimiento académico como medida cuasi-exclusiva de la calidad educativa en Chile. Posteriormente, se describen los efectos de esta gramática en el quehacer pedagógico y subjetividad de los estudiantes. En tercer lugar, explicaremos la metodología 
del trabajo. Tras esto, mostraremos los resultados del análisis los cuales finalmente se discutirán a la luz de la nueva política de inclusión educativa.

\section{Política del rendimiento académico y rendición de cuentas en Chile}

Desde la década de los años 80, el caso de Chile resulta emblemático en la promulgación de políticas educativas centradas en obtener los mejores rendimientos académicos de los alumnos, entendiendo a éste como un indicador central de la efectividad escolar. A nivel internacional, podemos encontrar programas homólogos como el impulsado por el gobierno de Bush conocido como No Child Left Behind. Bajo esta perspectiva se entiende que la efectividad escolar tiene como punto de partida el favorecer la igualdad de oportunidades de educación a estudiantes provenientes de distintos niveles socioeconómicos (ASSAEL et al., 2011; BELLEI, 2004; CORNEJO; REDONDO, 2007; RACZYNSKI; MUÑOZ, 2007). En este sentido, las escuelas pasan a ser las únicas responsables de propiciar una buena educación y calidad educativa para así disminuir las desigualdades sociales (RUIZSCHNEIDER, 2018).

Bajo esta perspectiva, se comienzan a implementar en educación una serie de cambios de tipo administrativo, financiero, así como también en torno a la evaluación de la calidad educativa. Al respecto, hay al menos tres aspectos que cambiarán paulatinamente la fisonomía de las escuelas y movilizarán un nuevo quehacer en los profesores, estudiantes, apoderados y en general, toda la comunidad educativa. Desde principios de los años 80, se inicia un proceso de descentralización y privatización de la educación, en el cual se permite la incorporación de proveedores privados al sistema educativo, con la consecuente coexistencia de establecimientos dependientes de las municipalidades, y otros pertenecientes a entidades privados (establecimientos particulares subvencionados), instalándose un cuasi mercado de escuelas, cada vez más diferenciados y autónomos, entendida como la forma de fomentar la competencia y por ende la calidad educativa (RANSON, 2008). En segundo lugar, en base a estos cambios se organiza la distribución de recursos mediante subsidios a la demanda, reconocido como voucher por alumno, lo que vendrá a reforzar el sistema educativo clientelar e individualista. Finalmente, en el corazón de estos cambios, en el año 1988, se erige como medida y evidencia de la calidad educativa una prueba estandarizada de competencias en matemáticas y lenguaje conocido como SIMCE (FALABELLA, 2014; CORNEJO, 2006; RACZYNSKI; MUÑOZ, 2007; SISTO, 2011). Cabe señalar, que bajo este contexto, adquiere relevancia el uso del libro de clases al interior de los establecimientos escolares, como instrumento cuya función consiste en identificar a estudiantes y padres; registrar la asistencia de los estudiantes y la actividad curricular, así como también, los comportamientos positivos o negativos de los estudiantes (LÓPEZ et al., 2019).

A pesar de estos cambios en pro de la eficacia educativa, a principios del año 2000 el gobierno de la concertación (centro izquierda) ve con preocupación que, a pesar del aumento significativo invertido en educación, los resultados en pruebas nacionales e internacionales mostraron un estancamiento, lo cual fue el terreno propicio para generar una reconfiguración del rol del Estado, mediante la profundización de lógicas empresariales al mundo educativo. 
La idea, fue mantener la privatización del sistema, pero incorporando instrumentos de evaluación y rendición de cuentas como forma de asegurar la calidad educativa (ASSAEL, et al., 2011). En este contexto se readecúa el sistema y objetivos curriculares para que éstos se alineasen con las mediciones realizadas por el SIMCE y el Programa para la Evaluación Internacional de Alumnos (PISA por su sigla en inglés).

Entre los años 2006 y 2011, de la mano de distintos movimientos sociales, fuertemente impulsados por estudiantes escolares, se abren nuevos debates en torno al financiamiento en educación, así como también en relación a lo que se entiende por calidad educativa, lo cual dio pie a una reforma. Dentro de esta reforma se promulgan nuevas leyes, entre ellas, la Ley de Subvención Educacional Preferencial (Ley 20.248, más conocida como SEP). Con esta ley se aumenta la subvención para los estudiantes más vulnerables del país, a cambio entre otras cosas, que las escuelas se comprometan al logro de mejoras en los resultados en el rendimiento de los estudiantes, medidos con la prueba estandarizada SIMCE (MURNANE; PAGE; VEGAS, 2009).

Bajo la ley SEP, las escuelas comienzan a ser evaluadas y clasificadas bajo las denominaciones de desempeño alto, medio, medio-bajo e insuficiente, dependiendo de los resultados obtenidos por los alumnos en la prueba SIMCE. De esta manera, se introduce a los ya conocidos estándares nacionales, otros sistemas de evaluación y clasificaciones escolares estandarizados, que están vinculados a las recompensas y sanciones individuales e institucionales (FALABELLA, 2014). En este contexto de reformas, en el año 2011 se promulga la Ley de Aseguramiento de la Calidad de la Educación (SAC) (Ley No 20.529), en el cual se suman al Ministerio de Educación dos nuevos organismos. Por una parte, se crea la Superintendencia de Educación Escolar, la cual está encargada de fiscalizar el cumplimento de las normativas educacionales y el buen uso de los recursos. Y, por otra parte, se crea la Agencia de Calidad de la Educación, la cual contempla dentro de sus funciones evaluar el desempeño de las escuelas, así como también orientar a aquellas que se encuentran por debajo de los estándares definidos. Con estos propósitos, dicho organismo clasifica todos los años a las escuelas en base a los desempeños del alumnado medidos en el SIMCE, instalándose la amenaza de cierre para aquellas escuelas que no salen de la categoría de insuficiente en el transcurso de cuatro años (ASSAÉL, et al., 2014).

\section{La gramática del rendimiento y sus efectos en los estudiantes}

Desde los discursos y políticas educativas que acabamos de mencionar, se ha instalado una gramática del rendimiento académico bajo el cual se sustenta el lema del mérito individual, en el cual se entiende que el esfuerzo individual y los buenos desempeños son el gran trampolín hacia el éxito en el futuro (LUNA-FIGUEROA, 2015). Esta gramática ha permeado de diferentes formas hasta el eslabón tradicionalmente más alejado de la práctica política: los propios estudiantes. Por ejemplo, como señala Peña (2010) se advierte que los niños y niñas construyen expectativas acerca de sus oportunidades a futuro basadas en su escolarización. Específicamente la autora da cuenta que los estudiantes, principalmente de escuelas municipalizadas, tienen puestas sus esperanzas en que una buena educación 
(entendida bajo el marco de la gramática meritocrática) les asegurará un mejor futuro, aspirando a un cambio radical en sus vidas, viendo de manera negativa su lugar de origen. En este sentido, la meritocracia cobra fuerza especialmente en contextos de pobreza, el cual, no solamente tienen repercusiones como el aumento de la competencia o el individualismo, sino que también en el miedo a ser evaluado, a no cumplir con los estándares, en definitiva, a no llegar a ser alguien (LANGER, 2018). El corolario de esta permeación se encuentra en la categoría que otros autores han denominado llegar a ser alguien, tener un buen futuro y servir para algo mediante el estudio y la profesionalización (MACHADO; MARTÍN; GRINBERG, 2016). Lo anterior ha conllevado a que los estudiantes vean reemplazada la lógica de aprender por la de lograr los mejores resultados (VILLALOBOS; QUARESMA, 2015), que como señala Carrasco (2014) estaría produciendo "sujetos respondedores de pruebas, uniformados, desprovistos de una experiencia educacional sustantiva" (CARRASCO, 2014, p. 9).

Así también, esta gramática del rendimiento ha tenido efectos en la segregación entre las escuelas y en la organización dentro del aula. Como señala Sisto y Fardella (2014) se ha dado una suerte de mercantilización de los alumnos, los cuales durante muchos años fueron seleccionados por las escuelas, en base a sus condiciones socioeconómicas y desempeños. En la práctica esto generó que los mejores estudiantes asistieran a escuela particulares o particulares subvencionadas, quedando la población más vulnerable en las escuelas municipales (RACZYNSKI; MUÑOZ, 2007), lo cual se está intentado revertir con la actual ley de inclusión (Ley 20.845), desde la cual se prohíbe la selección de los estudiantes. De igual manera, esta selección y segregación en base a los desempeños también se advierte al interior del aula, pues como señala Treviño, Villalobos, Vielma, Hernández y Valenzuela (2016), es posible advertir que su organización también se da en base al rendimiento académico de los estudiantes, y así, su posibilidad de producir resultados escolares positivos que resulten eficientes para el sistema educativo.

Finalmente, esta gramática del rendimiento, con sus correspondientes prácticas en el interior de las escuelas, ha implicado entender el proceso de enseñanza como una forma de control y así, el aprendizaje se ha transformado en una forma de obediencia (PEÑA, 2010). En este sentido, siguiendo a Foucault (1975), la práctica escolar deviene un proceso de subjetivación según el cual el mundo queda clasificado con términos marcados de lo que es sano/no sano, normal/ desviado, buen estudiante/mal estudiante, etc. Así, los propios estudiantes han pasado a ser vigías del comportamiento ajeno, definiendo quiénes son aptos o no, señalando aquellas conductas de los compañeros que son disruptivas para la consecución individual de la gran finalidad, que es lograr buenos desempeños (PEÑA, 2010). Por tanto, desde esta gramática se ha privilegiado un proceso de aprendizaje individual, orientado al control en el aula, que dificulta la emergencia de experiencias colaborativas de aprendizaje y de prácticas pedagógicas que sean relevantes para los estudiantes más allá de las calificaciones; así como también quedan obstaculizadas las experiencias de aprendizaje junto a compañeros, los vínculos con los docentes o la relación de confianza y apoyo entre pares, entre otros (ALBORNOZ; SILVA; LÓPEZ, 2015; PEÑA, 2010). 


\section{Método}

\section{Enfoque metodológico}

En este estudio se empleó una metodología de tipo cualitativo (WILLIG, 2008) que nos permitió comprender el mundo que construyen los estudiantes en los términos de sus propias palabras y acciones en relación a sus determinados contextos educativos (FLICK, 2014). En concreto, este trabajo se enmarca en un enfoque socioconstruccionista, desde el cual se entiende que el conocimiento se constituye en el intercambio social a partir del lenguaje (CABRUJA; IÑIGUEZ; VÁZQUEZ, 2000), que ordena las percepciones y hace que las cosas sucedan, dando cuenta de su carácter constructivo y creador de la interacción social y de diversos mundos sociales (POTTER; WETHERELL, 1987). A su vez, seguimos los planteamientos de la psicología discursiva como una aproximación metodológica, desde la cual nos permite reconocer las posiciones de los hablantes y las estrategias discursivas que se utilizan para construir versiones del mundo social (REYNOLDS; WETHERELL; TAYLOR, 2007).

\section{Participantes}

En el estudio participaron 59 adolescentes cuyas edades fluctúan entre los 12 y 15 años, pertenecientes a dos escuelas municipales de la región de Valparaíso, que corresponde a una de las regiones con mayor población del país. Dentro de los participantes, 19 son mujeres y 40 son hombres. En cada escuela se trabajó con el curso completo correspondiente a un séptimo grado. Dicha decisión se basó en evitar la selección arbitraria de los estudiantes, así como también, el poder acceder a las distintas voces que conforman un grupo natural como es un grupo curso.

Para realizar la investigación se solicitó la autorización a cada director/a de los establecimientos escolares, así como también se solicitó el consentimiento informado a cada uno de los padres y apoderado de los niños y niñas participantes. De igual manera, a los adolescentes se les hizo una presentación de los objetivos del estudio, momento en el cual se les invitó a participar voluntariamente, pudiendo participar finalmente sólo aquellos estudiantes que accedieran voluntariamente y que contaran con el consentimiento informado de los padres, madres o tutores. La carta de consentimiento y asentimiento informado, fueron aprobadas previamente por el Comité de Ética de la Pontificia Universidad Católica de Valparaíso.

\section{Producción de información}


Para la producción de información se utilizó la técnica denominada collaborative seeing and hearing (LUTTRELL, 2010; 2013), la cual sigue ciertos principios de lo que se conoce como fotovoz, especialmente en lo que refiere a propiciar un trabajo colaborativo entre los investigadores y participantes, que implica utilizar la creación de imágenes como medio de expresión de distintas realidades. Específicamente, se le solicitó a cada participante que sacaran fotografías de su vida cotidiana en la escuela. Posteriormente se utilizaron estas imágenes como dispositivo conversacional de la entrevista abierta, cuyo propósito fue considerar cómo los estudiantes elaboran discurso oral tras la presentación de sus imágenes fotográficas, aportando sus propias perspectivas y puntos de vista (SERRANO; REVILLA; ARNAL, 2016). Luego de realizar entrevistas individuales a cada uno de los participantes, se realizó un taller grupal participativo, que permitiera poner en discusión los hallazgos encontrados en la fase de entrevista individual. En términos investigativos, esta instancia permitió generar validación ecológica de la investigación, al compartir con los participantes los análisis y recibir retroalimentación por parte de ellos (WILLIG, 2008).

\section{Análisis de la producción de información}

Inserto en una investigación mayor, el corpus analizado para este artículo corresponde a los textos verbales producidos en las entrevistas individuales y en el taller grupal. El análisis de la información se realizó en dos fases. En un primer momento se realizó un análisis de contenidos temático (VÁZQUEZ, 1994), en la cual mediante una triangulación de técnicas se realizó una codificación abierta, categorización y reorganización de datos en torno a patrones recurrentes (FLICK, 2014), desde los cuales identificamos aquellas narrativas que permitían responder al objetivo del estudio. Al respecto se encontraron cuatro ejes narrativos, tal como se presentan en los resultados, cuyo análisis posterior se realizó considerando algunos recursos del análisis de discurso pragmático (POTTER; WETHERELL, 1987; WETHERELL; POTTER,1988) específicamente las estrategias discursivas y retóricas, tales como reiteraciones y metáforas (POTTER, 1998).

\section{Resultados}

Los resultados de nuestro trabajo han sido articulados en base a cuatro categorías principales que giran en torno al objetivo de analizar los efectos de esta gramática en estudiantes de sexto básico mediante las narrativas de su propia experiencia en el aula. Estos cuatro relatos son: 1) En el aula hay que ser buen estudiante; 2) No todos somos iguales en el aula; 3) La justificación de las sanciones en pro del rendimiento; y 4) No se puede perder tiempo con los otros. Dentro de los resultados se presentan extractos de los relatos de adolescentes, en los cuales la sigla No significa que el hablante es hombre, y la sigla Na, que la hablante es mujer. Así también, para referirnos la escuela, utilizamos la sigla E1 para la escuela 1, y la sigla E2 para la escuela 2. 


\section{En el aula hay que ser buen estudiante}

Esta primera categoría da cuenta de cómo los estudiantes, cuando hablan de su experiencia diaria en el aula, con frecuencia aluden al discurso en pro del rendimiento y las mejores calificaciones. En este sentido, la experiencia en el aula siempre aparece asociada a la importancia del rendimiento académico, específicamente aludiendo a la noción de buen estudiante, posicionándose entonces como el objetivo a alcanzar por parte de los estudiantes. El buen estudiante es indiscutiblemente aquel que tiene buenas calificaciones, siendo esta narración el eje central en torno al cual girará el resto de la argumentación sobre su experiencia en el aula, tal como se expresa en el taller participativo de la escuela 1:

No2: Necesitamos que haya menos bulla en la sala, que dejen de conversar constantemente, porque tenemos que aprender para sacarnos buenas notas para hacer alguien en la vida $E:$ ¿Tú crees que hay que sacarse buenas notas?

No2: Sí.

E: ¿Por qué?

No2: Porque si me saco buenas notas puedo ser alguien más.

E: y qué les pasa si se sacan malas notas

No2: Yo lo paso mal.

E: ¿Lo pasas mal por tus notas? ¿por qué crees que lo pasas mal?

No2: Es que cada vez que me dan como una mala nota, yo me pongo como triste.

$E$ : ¿Te pones triste? ¿y te pones triste por qué? ¿Te pones triste por ti, por los profes o por tu casa, tu familia?

No2: Por mí, porque lo estoy haciendo mal

E: Voy a tomar ese tema para el resto, ¿ustedes creen que las notas son importantes? ¿En esta escuela son importantes?

Todos: Si.

E: ¿Y por qué son importantes?

Na6: Para ser alguien en la vida (Taller participativo, E1)

El extracto anterior es muy ilustrativo respecto a la configuración del estudiante en el aula, así como también en las condiciones que se legitiman dentro de la sala de clases.

Para los estudiantes, desde temprana edad, la lógica del rendimiento ya aparece instalada en extractos como el anterior. En primer lugar, desde sus narrativas se configura como buen estudiante aquel que obtiene buenas calificaciones entendido como la única posibilidad de ser alguien en la vida. Es decir, para los estudiantes la búsqueda del mejor rendimiento y la persecución de ciertas formas de comportarse tiene una finalidad a futuro que les garantice conseguir mejores condiciones en la adultez (un mejor trabajo, mejor sueldo, tener más éxito, etcétera) y no en un resultado inmediato durante su trabajo diario en el aula. Esto es muy acorde a los mandatos internacionales sobre la calidad educativa, la cual está fuertemente reducida a una puntuación evaluada a través de pruebas estandarizadas tales como PISA y SIMCE. Hasta tal punto adquiere importancia el hecho de obtener una buena calificación, que los resultados en las evaluaciones inciden en sus afectos, puesto que el obtener una mala nota genera afectos de tristeza, así como la autocalificación de ser un mal estudiante, 
adoptando toda la lógica del remordimiento propia del capitalismo actual (HAN, 2018). Así también, en sus relatos se advierte que se legitima sólo una forma de estar dentro de la sala de clases, la cual es en silencio (con menos bulla), esto, como la forma que les posibilita poder escuchar al docente, lo que a su vez, redunda en su buen rendimiento.

2. No somos todos iguales en el aula.

Cuando la lógica de ser buen estudiante, entendida como tener buenas calificaciones y tener un comportamiento disciplinado, se asienta como la forma en la cual se debe ser y estar en el aula, se propicia la emergencia de distinciones entre tipos de estudiantes, aludiendo a que no todos son buenos estudiante, pues no todos obtienen buenas calificaciones y se comportan de la forma esperada en este espacio. Al respecto, encontramos el binomio de estudiante inteligente versus el estudiante flojo, como parte de las narraciones que se constituyen desde la gramática del rendimiento académico que acabamos de ver. Así se muestra en el taller participativo de la escuela 2, tal como se señala:

No1: Por ejemplo, yo digo los flojos y los inteligentes, los flojos también se pueden reflejar en las notas, en la motivación, por ejemplo, el Carlos cuando le piden algo, no es por ejemplo como el Sebastián que va a decir "ah pucha".

No2: Para mí las personas inteligentes son como los más responsables, y las flojas son como los que no les dan ganas de hacer nada.

No15: Yo creo que en vez de inteligentes y flojos, son responsables con irresponsables (Taller participativo, E2)

La consecuencia de que no todos los estudiantes son iguales, pues no todos obtienen buenas notas, así como tampoco se comportan adecuadamente dentro de la sala de clases, cae por su propio peso en base a estos fragmentos narrativos. De esta forma, los estudiantes comienzan a narrar diferentes clasificaciones de sus compañeros a raíz del mandato educativo, legal e institucional del buen rendimiento que describimos al inicio. De este modo, se configuran dicotomías entre los estudiantes flojos e inteligentes; responsables e irresponsables, etcétera, como categorías organizadoras del mundo social del aula con las que los estudiantes ya dan cuenta de sus compañeros.

De esta manera, la lógica del desempeño penetra hasta el interior de los estudiantes, de niñas y niños de doce años que ya entienden el mundo y a sí mismos bajo la óptica de las buenas notas para ser alguien en la vida, estableciendo comparaciones con aquellos compañeros que lo hacen mejor frente a aquellos con más dificultades. Por tanto, desde esta distinción no sólo se clasifica a los compañeros, sino que el propio estudiante también queda clasificado, lo que conlleva a la justificación y normalización de las prácticas punitivas de aulas y pasillos hacia los "malos estudiantes", que son aquellos que conversan, los que no están quietos en el aula y tienen peor rendimiento, como revisaremos en la siguiente categoría. 


\section{La justificación de las sanciones en pro del rendimiento.}

Como acabamos de ver, la búsqueda del rendimiento conlleva directamente la clasificación de los estudiantes entre buenos y malos, es decir, entre aquellos capaces de conseguir ese rendimiento para ser alguien en la vida y los que no. Esta categorización a los compañeros y a uno mismo en función de sus calificaciones y comportamiento contribuye a la emergencia de una narrativa de justificación del castigo y de las prácticas punitivas para los malos estudiantes, pues dificultan el rendimiento de los demás. De este modo, los estudiantes hablan de forma normalizada y justificada acerca del castigo hacia los compañeros y hacia sí mismo, como forma de asegurar las buenas calificaciones, tal como se señala:

E: ¿y qué te pasa con esto? (refiriéndose a la bulla en la sala)

Na: como estoy al último, ni callada puedo escuchar bien porque siempre

hablan muy bajo entonces eso como que me... frustra un poco porque a

veces me va mal en las pruebas porque no escucho.

E: ¿Molestando en qué- de qué manera?

No: Por ejemplo... gritando a veces

E: $\mathrm{Hm}$

No: Corriendo por la sala... hablando mientras el profe habla, así

E: ¿Y a esos alumnos qué les, qué les hacen?

No: Los retan no más

E: Ya, ¿no los sacan de la sala?

No: O sea, hay veces que los sacan de la sala, que no-que los mandan a la biblioteca

E: Ya, hmhm, ¿y a ti qué te parece eso?

No: O sea hm, que yo opino que está bien, que- si ellos están molestando que los saquen porque mientras ellos estén molestando, los demás no van a poder aprender bien (Participante 3, escuela 1).

En el caso particular de este relato, los estudiantes justifican el tipo de ambiente que debe reinar en la sala: el silencio, la calma, el orden. La justificación de este orden nuevamente persigue el fin de servir para no obtener malas calificaciones y para aprender bien, y si este fin no se consigue, los estudiantes justifican la sanción a los compañeros en forma de expulsión del aula, o ser retados.

Pero, de la misma forma que ocurría en la categoría anterior, donde los adolescentes se entendían a sí mismos como malos estudiantes (por su notas o comportamiento), a la hora de narrar la justificación de las prácticas punitivas, también justifican y naturalizan que, por tanto, ellos mismos reciban castigos cuando hablan o no se comportan de la forma esperada:

E: Ah. ¿Y qué te parece que te, que te haya echado?

No: Yo lo encontré como justo porque es verdad que yo estaba hablando, yo estaba riéndome en una prueba porque estaba, o sea, yo me estaba riendo solo por una cues, porque me acordé de una cosa y la tía me echó 
E: Mm. Y te parece justo.

No: La, el primer día sí, el segundo día que simplemente entré a clases

ella me echó (Participante 24, escuela 1).

Por tanto, la narrativa en torno al buen estudiante no solamente interpela a las prácticas que pueden favorecer su rendimiento, sino también a aquellas que son aceptables de llevar a cabo en caso de un mal estudiante que dificulta la obtención de buenas calificaciones a los demás. Así, otras alternativas que formarían parte de una lógica formativa, tales como la enseñanza de otros comportamientos que no entorpezcan a los compañeros, o la búsqueda de pedagogías donde sea compatible hablar, levantarse, trabajar en grupo... quedan invisibilizadas bajo este argumento.

\section{No se puede perder tiempo con los otros}

Pero pese a la justificación y normalización de las prácticas punitivas y la internalización del discurso del rendimiento, los propios estudiantes narran la expectativa de prácticas educativas alternativas a la del orden y el silencio en el aula: espacios y pedagogías donde pueden trabajar en grupo, levantarse del asiento, hablar con los compañeros, etcétera.

\section{Me gustaría hacer actividades en grupo, porque todos pueden estar ahí, contando su opinión de algo...podría ser más entretenido estar en clases y podríamos compartir nuestros aprendizajes (Participante 9, niño, E1).}

El aprender por aprender es vinculado por los adolescentes a espacios de aprendizaje donde pueden trabajar colaborativamente, de forma dinámica o por otros medios más allá de la tradicional clase de cátedra donde el profesor transmite conocimientos de forma unidireccional al estudiante. Sin embargo, este interés por trabajar con los otros y en equipo sigue estando subordinado a que fruto de la colaboración o de la dinámica pedagógica alternativa, se consiga un buen rendimiento:

No: Más veces, nos hacen trabajar en parejas, como nos sientan de a dos, nos hacen trabajar en parejas

E: ¿Y qué te parece tener que trabajar de a dos? ¿cómo ha sido tu experiencia en eso?

No: Eh buena

E: $Y a$

No: O sea generalmente cuando me hacen trabajar con alguien que sí se empeña en trabajar

E: Hmhm

No: Eh trabajo con él, pero con alguien como que no le interesa, trabajo solo, aunque nos hagan trabajar juntos yo trabajo solo

[...] E: Ya, ¿y cómo tomaste esa decisión?

No: Porque... era como ya, si él no quiere trabajar pa' qué voy a esperar pa' que él de verdad le ponga empeño, si igual voy a estar perdiendo el tiempo (participante 29, imagen 2, niño E1) 
Pese a que los estudiantes reconocen que es más atractivo trabajar de forma grupal o de otros modos a la clásica pedagogía unidireccional del profesor, los estudiantes supeditan estas alternativas pedagógicas a la consecución de un buen rendimiento. Hasta tal punto que los estudiantes solo aceptan trabajar en grupo si el compañero o compañera no les hace perder el tiempo, perderlo en conseguir buenas notas para ser buen estudiante y así ser alguien en la vida en el futuro. De esta forma, participantes refuerzan el argumento del buen estudiante como aquel que obtiene buenas calificaciones, pues será con él o ella con los únicos con los que quieren trabajar colaborativamente pese a reconocer que este tipo de instancias son más divertidas o interesantes.

\section{Discusión}

En este estudio hemos dado cuenta de las narrativas de las que los estudiantes dan cuenta sobre su experiencia en el aula. En ellas, la gramática del mérito impulsada por organismos internacionales como la OCDE o el banco mundial, y también por diferentes instituciones nacionales tales como el MINEDUC y la Agencia de calidad, permea hasta el lugar más íntimo y frágil del sistema educativo, como es la subjetividad de los estudiantes. Si bien las categorizaciones entre los distintos estudiantes se pueden encontrar en los orígenes del aula (DUSSEL \& CARUSO), hoy se advierte que son los propios estudiantes quienes anteponen el mérito a cualquier otro aspecto, entendiendo al buen estudiante como aquel que consigue buenas notas y se comporta de ciertas formas muy particulares (sentado, sin hablar, sin molestar). En este sentido, esta gramática permea en los estudiantes, hasta el punto de justificar el castigo para aquellos que denominan como malos estudiantes, que son aquellos que molestan o no obtienen las mejores notas; incluso cuando el mal estudiante es uno mismo. En definitiva, de estos resultados, emerge la narrativa general del buen estudiante.

Los resultados de nuestro estudio dan cuenta de cómo la política general de los últimos cuarenta años en Chile, y en particular la política educativa no sólo ha llegado a repercutir en los estudiantes como usuarios y clientes finales de la misma; sino que ha calado hasta su propia subjetividad, estableciendo una forma muy concreta y particular de que los niños de doce años se entiendan a sí mismos y a sus pares dentro del aula. Este entendimiento de sí y de los demás se caracteriza por la fetichización del mérito y sus altas consecuencias, entendido como la persecución de la mayor calificación posible en las pruebas y evaluaciones a las que se enfrentan, indistintamente de que esto suponga un perjuicio para aquellos compañeros que les impiden llegar a las mejores calificaciones. En este sentido, se replica desde las narrativas de los estudiantes el binomio instalado desde las políticas educativas entre los estudiantes aptos y no aptos (FALABELLA, 2014), siendo los primeros los que responden académicamente, y los segundos, los que no se adaptan conductualmente, así como tampoco rinden en los términos esperados. 
Además, este culto a las buenas notas cala también en ellos mismos, lo cual supone la justificación y normalización de la sanción incluso hacia sí mismo en caso que considere que él o ella no obtiene esas buenas calificaciones, o si es un estudiante poco disciplinado (el que habla, el que no se sienta, el que sale de clase...). Así, la instalación de la cultura de la performatividad y de la rendición de cuentas se revela en el comportamiento de los los estudiantes durante las actividades cooperativas, porque ellos y ellas tienden a adoptar un esquema de trabajo más competitivo que colaborativo y perciben a sus pares más como amenazas para su desempeño individual que como soporte en su proceso de aprendizaje (LUNA-FIGUEROA, 2015). Pero esta narración del buen estudiante de los y las estudiantes, no sólo tiene un amparo en sus relatos, sino que tiene un sustento real y material en las dinámicas escolares cotidianas que configuran el comportamiento de los niños y sus relaciones. Por ejemplo, la única forma de optar a establecimientos emblemáticos, considerados los de mejor nivel y los que más oportunidades laborales darán a los estudiantes, es mediante un expediente académico y un comportamiento intachable. Pese a que esta lógica está cambiando paulatinamente (por ejemplo, con la Ley de Inclusión), aún predomina puesto que imperan otros instrumentos de selección que se centran también en calificaciones con la idea de que hay que ser buen estudiante, como el libro de clases -el instrumento de evaluación de buena conducta en Chile- (LÓPEZ et al., 2019), y el propio SIMCE (FALABELLA, 2014). Es posible plantear que estos instrumentos de selección y el tipo de comportamiento performativo que dirigen -ser excelente alumno, evidenciado a través de las notas; y tener una conducta intachable, evidenciado mediante la ausencia de observaciones negativas en el libro de clasesgeneran un escenario de altas consecuencias para los estudiantes, especialmente para aquellos que quedan situados en los extremos de este "estudiante ideal": por una parte, aquellos estudiantes con talentos académicos en sectores de pobreza que buscan (ello y/o sus padres) activamente la movilidad social y que arriesgan no quedar seleccionados en los liceos emblemáticos; y por otra parte, los estudiantes con muy bajo rendimiento y/o altos problemas de conducta quienes arriesgan ingresar al ciclo de expulsión o amenaza de expulsión que comienza con prácticas disciplinares punitivas como consecuencia de tener muchas anotaciones negativas: ser enviado a inspectoría, a la oficina del director/a, ser suspendido, entre otras.

En los últimos años, se advierte que esta gramática del mérito se ha visto cuestionada tanto por ciertas leyes y normativas en el ámbito general, como por prácticas locales; que tratan de promover una mayor inclusión y reconocimiento a la diversidad de los estudiantes y de las escuelas, tal es el caso de la Ley 20.845, promulgada en Chile en el año 2016, la cual dentro de sus normativas prohíbe la selección en las escuelas, como una forma de promover la diversidad social y académica junto con potenciar la educación pública, así como también el poder avanzar hacia escuelas más democráticas (VALENZUELA; MONTECINOS, 2017). Sin embargo, estos intentos, se contradicen con las prácticas derivadas del SAC, el cual a través de la Agencia de Calidad evalúa a la escuela en base al rendimiento académico, por lo que vemos que existe un cuestionamiento de fondo de la gramática meritocrática, en la que se apueste por 
indicadores que midan otras variables (socioemocionales, por ejemplo), por lo que se sigue atados a la lógica de la estandarización y a las altas consecuencias. El ejemplo de esto lo encontramos en las narrativas de los estudiantes, los cuales siguen narrando su experiencia en el aula desde la lente del mérito. Esto supone una tensión entre lo que la política educativa enuncia en la teoría, y lo que finalmente supone, lo que nos desafía a encontrar modos sobre cómo ir relevando o generando una narrativa alternativa a la del mérito, cuya centralidad sea un modelo realmente inclusivo que reconozca como pilar central el acceso a una educación de calidad y el reconocimiento de todos los individuos que la hacen posible sin que el mérito, la competitividad o la individualización ocupen una posición remarcable. Por ejemplo, una alternativa es la propuesta por Langer (2011), quien reflexiona en torno a las formas de resistencia en el plano educativo, en lo que denomina comunidades pedagógicas, reinventando el significado original de las comunidades pre-liberales a las características de las sociedades del gerenciamiento. Se trataría de comunidades atravesadas por la autogestión y menos sujetadas por las instituciones; con pocos recursos, pero distribuidos y manejados por el bien del conjunto (y no de las personas individuales), y donde el objetivo final no es la competencia y el mérito sino más bien la autorregulación de las conductas y el desarrollo de habilidades como la crítica, la justicia o la participación. Otro caso lo encontramos en la propuesta de Vásquez (2010) quien propone que se incluya los valores y las prácticas relacionadas con el cuidado como centro del quehacer en la escuela, entendida como condición imprescindible para universalizar la ética de la justicia, que permitan trascender ciertas dicotomías, que sitúan como pares opuestos ya sea lo masculino y lo femenino, lo racional y lo emotivo, o a los más o menos inteligentes, en pro de fortalecer una educación ciudadana que permita el desarrollo integral de los estudiantes y promuevan su convivencia con el otro.

Queda pendiente por tanto la creación de dispositivos que permitan la emergencia de otras subjetivaciones y narraciones de la experiencia en el aula, para lo cual es necesario articular un nuevo enfoque y nuevos discursos que sean transversales al sistema educativo y cuya centralidad sea volver a la reflexión de lo pedagógico y ya no lo burocrático o el seguir saturando el aparato administrativo de la educación: desde las políticas nacionales hasta las prácticas cotidianas de los estudiantes en las aulas, pasando por otros documentos y textos culturales y sociales; donde el interés y las capacidades de los estudiantes junto a la experiencia de los profesores, sean elementos protagónicos. Sólo de esta forma se podrá plantar la semilla de narraciones, experiencias y prácticas sociales y ciudadanas donde el otro sea parte reconocida de la sociedad independientemente de sus valores, ideología, capacidades, nacionalidad o cultura.

\section{Referencias}


ALBORNOZ, Natalia; SILVA, Natalia; LÓPEZ, Mauricio. Escuchando a los niños: Significados sobre aprendizaje y participación como ejes centrales de los procesos de inclusión educativa en un estudio en escuelas públicas en Chile. Estudios Pedagógicos, v. XLI, n. especial, p. 81-96, 2015.

ANDERSON, Stephen E. et al. No one way: differentiating school district leadership and support for school improvement. Education as Change, v. 13, n. 4, p. 403 - 430, 2012. https://doi.org/10.1007/s10833012-9189-y.

ASSAÉL, Jenny et al. La empresa educativa chilena. Educ. Soc., Campinas, v. 32, n. 115, p. 305 - 322, 2011.

ASSAÉL, Jenny et al. Transformaciones en la cultura escolar en el marco de la implementación de políticas de accountability en Chile. Un estudio etnográfico en dos escuelas clasificadas en recuperación.

Estudios Pedagógicos, v. 40, n. 2, p. 7 - 26, 2014. doi: http://dx.doi.org/10.4067/S0718$\underline{07052014000300001}$

BALL, Stephen John. The teacher's soul and the terror of performativity. Journal of Education Policy, v. 18 , n. 2, p. $215-228,2003$.

BALL, Stephen John. Educational reform, market concepts and ethical re-tooling. En: S. J. BALL. Education Policy and Social Class. The selected works of Stephen Ball. London, NY: Routledge, 2006.

BELLEÏ, Cristian. Equidad Educativa en Chile: un Debate Abierto. Serie reflexiones: Infancia y adolescencia n². Santiago-Chile: UNICEF, 2004.

CABRUJA, Teresa; ÍÑIGUEZ, Lupicinio; VÁZQUEZ, Félix. Cómo construimos el mundo: relativismo, espacios de relación y narratividad. Análisi v. 25, p. 61 - 94, 2000.

CARRASCO, Alejandro. Mecanismos performativos de la institucionalidad educativa en Chile: pasos hacia un nuevo sujeto cultural. Observatorio cultural, v. 15, p. 4-10, 2014. Recuperado desde http://www.observatoriocultural.gob.cl/revista/2-articulo-1/15-mecanismos-performativos-de-lainstitucionalidad-educativa-en-chile-pasos-hacia-un-nuevo-sujeto-cultural-2/

DUSSEL, Inés; Caruso, Marcelo. La invención del aula: Una genealogía de las formas de enseñar. Buenos Aires, Argentina: Ediciones Santillana, 2006.

CORNEJO, Rodrigo; REDONDO, Jesús María. Variables y factores asociados al aprendizaje escolar: una discusión desde la investigación actual. Estudios pedagógicos (Valdivia), v. 33, n. 2, p. 155-175, 2007.

FALABELLA, Alejandra. The Performing School: The Effects of Market y Accountability Policies. Education Policy Analysis Archives, v. 22, n. 70, 2014. http://dx.doi.org/10.14507/epaa.v22n70.2014.

FALABELLA, Alejandra; OPAZO, Catalina. Sistema de Aseguramiento de la Calidad y procesos de mejoramiento: una mirada desde la gestión educativa. Informe Ejecutivo. Santiago de Chile: Universidad Alberto Hurtado, 2014.

FARDELLA, Carla; SISTO, Vicente. Nuevas regulaciones del trabajo docente en Chile. Discurso, subjetividad y Resistencia. Psicología \& Sociedade, v. 27, n.1, p. 68 - 79, 2015.

FOUCAULT, Michel. Vigilar y Castigar. El nacimiento de la prisión. Madrid, España: Siglo XXI editores.

FLICK, Uwe. An Introduction to Qualitative Research. London: Sage. 2014.

GEWIRTZ, Sharon. The managerial school: postwelfarism and social justice in education. London: Routledge, 2002.

GEWIRTZ, Sharon.; BALL, Stephen. From 'Welfarism' to 'New Managerialism': Shifting discourses of school headship in the education Marketplace. Discourse: Studies in the Cultural Politics of Education, v. 21, n. 3, 253 - 268, 2000. doi: 10.1080/713661162 
GRINBERG, Silvia. Educación, Gubernamentalidad y Después... la configuración de una nueva pastoral. En CORTÉS, Ruth y Marín, Dora (comps) Gubernamentalidad y Educación. Discusiones Contemporáneas. Bogotá: IDEP, 2011. p. 61 - 80.

GUNTER, Helen. Policy and Workforce Reform in England. Educational Management Administration \& Leadership, v. 36, n. 2, p. 253-270, 2008. doi: 10.1177/1741143207087776.

HAN, Byung-Chul. La sociedad del cansancio. Barcelona: Herder, 2018.

LANGER, Eduardo. Comunidades Pedagógicas Emergentes en la Argentina del Siglo XXI. En: Cortés, Ruth y Marín, Dora (Comps) Gubernamentalidad y educación. Discusiones contemporáneas. Bogotá: IDEP, 2011. p. $61-80$.

LANGER, Eduardo. Los Miedos En Tiempos De Crisis Según Docentes Y Estudiantes De Argentina. Universitas, v. 28, p. $19-38,2018$.

LÓPEZ, Verónica et al. Traduciendo las Políticas de Convivencia Escolar: Análisis de Dispositivos Sociotécnicos en Argentina y Chile, Psicoperspectivas, v. 18, n.1, p.1-15, 2019.

LUNA-FIGUEROA, Laura. Construyendo "la identidad del excluido": Etnografía del aprendizaje situado de los niños en una escuela básica municipal de Chile. Estudios Pedagógicos, v. 61, n especial, p. 97-113, 2015.

LUTTRELL, With. A camera is a big responsibility: A lens for analyzing children's visual voices, Visual Studies, v. 25, n. 3, p. $224-238,2010$.

LUTTRELL, With. Children's Counter-narratives of Care: Towards Educational Justice. Children \& Society, v. 27, p. 295 -308, 2013. doi:10.1111/chso.12033

MACHADO, Mercedes; MARTÍN, Luciano; GRINBERG, Silvia. Relatos de Infancias: Nacer y Vivir en las Villas del Sur Global. Cartografía y Devenir de la Subjetividad en las Sociedades Contemporáneas. Última Década, v. 24, n. 45, p. 140 - 157, 2016.

MINISTERIO DE EDUCACIÓN, Chile. Ley 20.248, de 1 de febrero de 2008. Establece Ley de Subvención Escolar Preferencial. Diario Oficial de la República de Chile, 2008. En: http://bcn.cl/1uv4p.

Ley 20.529, de 27 de agosto de 2011. Sistema Nacional de Aseguramiento de la calidad de la Educación Parvularia, Básica y Media y su Fiscalización. Diario Oficial de la República de Chile. En: http://bcn.cl/1uv5c.

Ley 20.845, de 8 de junio de 2015. De Inclusión Escolar Que Regula La Admisión De Los Y Las Estudiantes, Elimina El Financiamiento Compartido Y Prohíbe El Lucro En Establecimientos Educacionales Que Reciben Aportes Del Estado. Diario Oficial de la República de Chile. En: http://bcn.cl/1uv1u.

MURNANE, Richard; PAGE, Lindsay; VEGA, Emiliana. Distribución de los rendimientos estudiantiles en Chile. Análisis de línea base para la evaluación de la subvención escolar preferencial (SEP). 2009. Recuperado de https://docplayer.es/54002251-Distribucion-de-los-rendimientos-estudiantiles-en-chileanalisis-de-linea-base-para-la-evaluacion-de-la-subvencion-escolar-preferencial-sep.html

NIESCHE, Richard. Governmentality and My School: School Principals in Societies of Control. Educational Philosophy and Theory v. 47, n. 2, p. 133-145, 2015. Doi: http://dx.doi.org/10.1080/00131857.2013.793925

PEÑA, Mónica. ¿Quién es el Niño? Revisión y análisis de algunos conceptos teóricos relevantes para el acercamiento pedagógico a la infancia en Chile. Revista de Estudios y Experiencias en Educación, v. 1, n. 5 , p. $75-88,2004$. 
PEÑA, Mónica. Hacia una recuperación de la subjetividad en el proceso de conocer en el contexto escolar: La pregunta por el saber en niños y niñas de Educación Básica Chilena. Estudios Pedagógicos, v. 36, n. 2, p. $195-211,2010$.

POTTER, Jonathan. La representación de la realidad. Barcelona, España: Paidós, 1998.

POTTER, Jonathan; WETHERELL, Margaret. Discourse and Social Psychology. London: Sage, 1987.

RACZYNSKI, Dagmar; MUÑOZ, Gonzalo. Reforma Educacional Chilena: El Difícil Equilibrio entre la Macro y Micro Política. Revista Electrónica Iberoamericana sobre Calidad, Eficacia y Cambio en Educación, v. 5, n. 3, pp. 40-83, 2007.

RANSON, Stewart. Public accountability in the age of neo-liberal governance. Journal Education Policy, v. 18, n. 5, p. 459-480, 2003. Doi: https://doi.org/10.1080/0268093032000124848.

RANSON, Stewart. The Changing Governance of Education. Educational Management Administration y Leadership, v. 36, n. 2, p. 201-219, 2008. doi: 10.1177/1741143207087773

REYNOLDS, Jill; WETHERELL, Margaret; TAYLOR, Stephanie. Choice and chance: Negotiating agency in narratives of singleness. The Sociological Review, v. 55, p. 331 - 351, 2007. doi:10.1111/j.1467954X.2007.00708.x

RUIZ-SCHNEIDER, Carlos. Educación y plítica en la transición Chilena. En RUÍZ-SCHNEIDER, Carlos; REYES, Leonora; HERRERA, Francisco (eds). Privatización de lo público en el sistema escolar. Santiago-Chile: LOM ediciones. 2018.

SERRANO, Araceli.; REVILLA, Juan Carlos; ARNAL, María. Narrar con imágenes: entrevistas fotográficas en un estudio comparado de "resiliencia" social y resistencia ante la crisis. EMPIRIA. Revista de Metodología de Ciencias Sociales, v. 35, p. 71-104, 2016. DOI5.2016.17169

SISTO, Vicente. Nuevo profesionalismo y profesores: una reflexión a partir del análisis de las actuales políticas de 'profesionalización' para la educación en Chile. Signo y Pensamiento, v. XXXI, p. 178-192, 2011. Recuperado de http://www.redalyc.org/articulo.oa?id=86022458013

SISTO, Vicente. Inclusión "a la Chilena": La inclusión escolar en un contexto de políticas neoliberales avanzadas. Archivos Analíticos de Políticas Educativas, v. 27, n. 23, 2019. http://dx.doi.org/10.14507/epaa.27.3044

SISTO, Vicente; FARDELLA, Carla. El eclipse del profesionalismo en la era de la Rendición de CuentasModelando Docentes en el contexto del Nuevo Management Público. Cadernos de Educação, n. 49, 323, septembro/dezembro, 2014.

TERRENI, Luciana; GRINBERG, Silvia; PEREZ, Andrés. Distribución y acceso a la cultura en las sociedades del gerenciamiento a través de las nuevas tecnologías. Un estudio de caso en una escuela secundaria de Gualeguaychú, Entre Ríos. Informes Científicos Técnicos- UNPA, v. 9, n. 3, p. 40 - 64, 2017.

TREVIÑO Ernesto et al. Trayectorias escolares de los estudiantes y agrupamiento al interior del aula en los colegios chilenos de enseñanza media. Análisis de la heterogeneidad académica al interior de las escuelas. Pensamiento Educativo. Revista de Investigación Educacional Latinoamericana, v. 53, n. 2, p. 1 - 17, 2016. doi: 10.7764/PEL.53.2.2016.5.

VALENZUELA, Juan Pablo; MONTECINOS, Carmen. Estructural reforms and equity in Chilean schools, Oxford Research Encyclopedia of Education. 2017. doi: 10.1093/ ACREFORE/9780190264093.013.108.

VÁZQUEZ, Félix. Análisis de contenido categorial: el análisis temático. Barcelona: Universidad Autónoma de Barcelona. 1994. 
VÁZQUEZ, Victoria. La perspectiva de la ética del cuidado: una forma diferente de hacer educación. Educación XXI, v. 13, n. 1, p. 177 - 197, 2010.

VILLALOBOS, Cristóbal y QUARESMA, María Luisa. Sistema escolar chileno: características y consecuencias de un modelo orientado al mercado. Convergencia UAEM, n. 69, septiembre diciembre, p. $63-84,2015$.

WILLIG, Carla. Qualitative Research Design. In Willig, Carla (ed.). Introducing Qualitative Research in Psychology: Adventures in Theory and Method. United Kingdom: Mc Graw Hill, p. 15 - 32, 2008.

WHETHERELL, Margaret; POTTER, Jonathan. El análisis del discurso y la identificación de repertorios interpretativos. En GORDO, Angel; LINAZA, José (coord.). Psicologías, Discursos y Poder (PDP). Madrid: Visor. 1998.

\section{Correspondência}

Lorena Ramírez-Casas del Valle: Centro de Investigación para la Educación Inclusiva, Pontificia Universidad Católica de Valparaíso.

E-mail: lorena.ramirez@pucv.cl

Orcid: https://orcid.org/0000-0001-5746-7777

Enrique Baleriola: Universitat Oberta de Catalunya.

E-mail: ebebaes@gmail.com

Orcid: https://orcid.org/0000-0003-2899-6316

Vicente Sisto: Escuela de Psicología, Centro de Investigación para la Educación Inclusiva, Pontificia Universidad Católica de Valparaíso.

E-mail: vicente.sisto@pucv.cl

Orcid: https://orcid.org/0000-0003-4510-4041

Verónica López: Escuela de Psicología, Centro de Investigación para la Educación Inclusiva, Pontificia Universidad Católica de Valparaíso.

E-mail: veronica.lopez@pucv.cl

Orcid: https://orcid.org/0000-0001-7405-3859

Felipe Aguilera: Escuela de Psicología, Centro de Investigación para la Educación Inclusiva, Pontificia Universidad Católica de Valparaíso.

E-mail: aguilera.FI@hotmail.com

Orcid: https://orcid.org/0000-0002-9489-2139 


\section{Agradecimentos}

Este trabajo contó con el apoyo del Programa PIA-CONICYT - Proyecto CIE 160009. A su vez, este trabajo cuenta con apoyo del proyecto FONDECYT REGULAR N 1191015 y N 1191267 y Beca N²1190891

Doctorado Nacional, del Consejo Nacional de la Ciencia y la Tecnología de Chile (CONICYT).

Texto publicado em Currículo sem Fronteiras com autorização dos autores. 